Results We identified four draft key drivers to: (1) address social determinants of health during prenatal, delivery, and postpartum care; (2) utilize race/ethnicity medical record and quality data; (3) engage patients, birth partners, and communities; and (4) engage and educate providers and nurses, to improve birth equity. See the Table 1 for the working version of key drivers and corresponding strategies and measures.

Conclusions The ILPQC Birth Equity Initiative will be further developed with input from the initiative clinical leads and stakeholders for implementation with up to the 117 birthing hospitals in IL starting in 2021. ILPQC and hospital teams will monitor progress on initiative measures monthly to inform statewide improvement efforts and evaluate the implementation approach for potential replicability in other states.

\section{UNDERSTANDING INSTITUTIONAL PHYSICIAN CHRONIC OPIOID PRESCRIPTION PRACTICES FOR THE IMPROVED IMPLEMENTATION OF NEWLY DEVELOPED EMR TOOLS}

Sonal Gagrani, Enas Kandil. University of Texas Southwestern Medical Center, USA

\subsection{6/bmjoq-2020-IHI.25}

Background Chronic non-cancer pain (CNCP) affects a significant portion of the United States population each year and is often treated with chronic opioids. Several policies including the 2016 'CDC Guidelines for Prescribing Opioids for Chronic Pain' have been developed to guide CNCP management. These recommendations include the use of pain management agreements, urine drug screening, prescription monitoring programs (PMP), and risk and pain assessments among others. In Texas, several recent policies have made

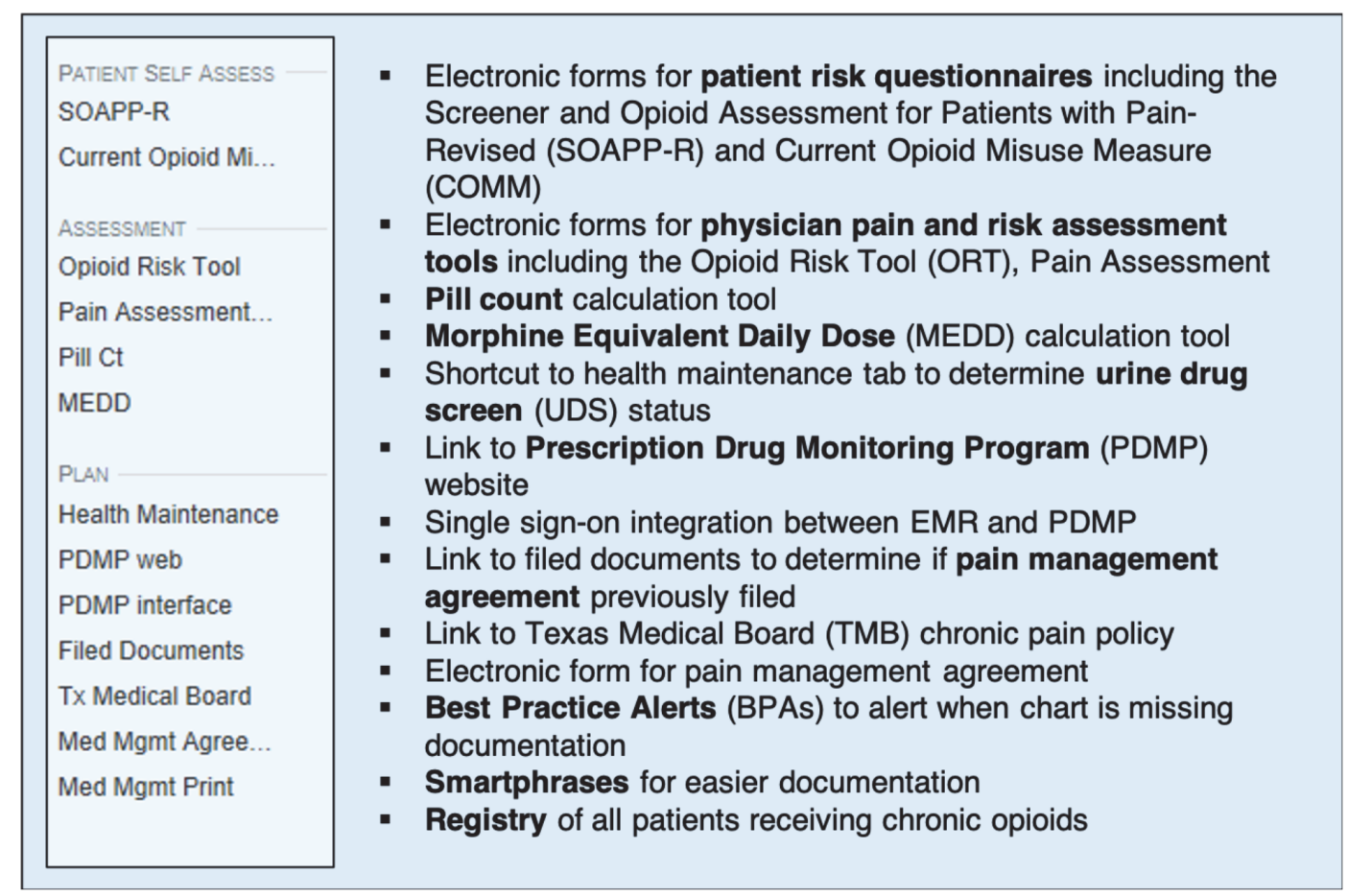

Abstract 25 Figure 1 Summary of EMR chronic paint navigator tool function and other EMR-integrated tools.

A

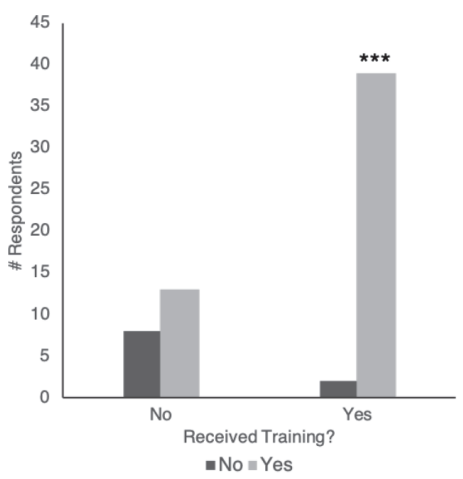

B

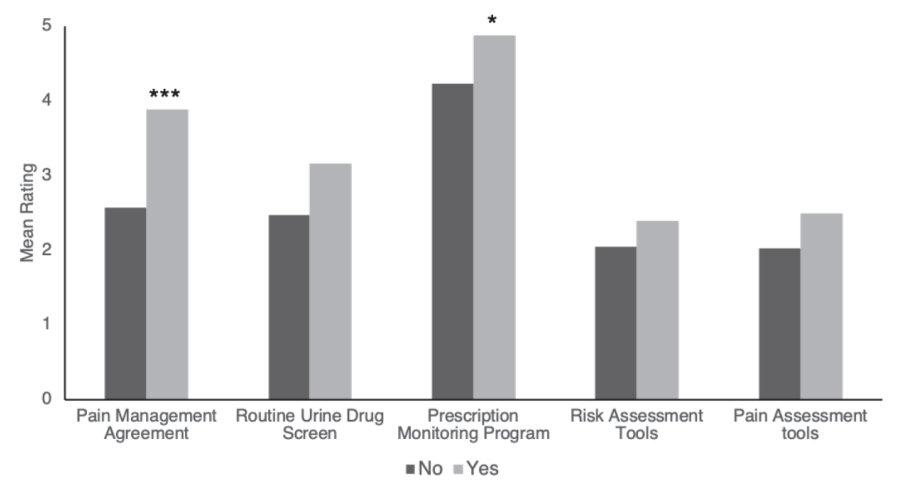

Abstract 25 Figure 2 Intervention efficacy, A) Reported awareness of CNCP policy based on training exposure, B) Reported use of guideline treatment components with and without intervention use, ${ }^{* * *} p<.0001,{ }^{*} p<.05$ 
Abstract 25 Table 1 Summary of survey respondent characteristics pre- and post- intervention with cancer and palliative care respondents removed, $S \mathrm{SD}=$ standard deviation

\begin{tabular}{lcc}
\hline & Pre-intervention, \% (No.) & Post-intervention, \% (No.) \\
\hline $\begin{array}{l}\text { Years practicing medicine in } \\
\text { Texas, mean (SD) }\end{array}$ & $14.58(12.12)$ & $14.31(10.97)$ \\
Years practicing medicine \\
overall, mean (SD)
\end{tabular}

Abstract 25 Table 2 Distribution of other chronic pain policy recommendations, \% (No.) Measured on a scale from 1-5 where $1=$ Never, 2 = Rarely, 3 = Sometimes, $4=$ Often, $5=$ Very Often. MEDD $=$ Morphine Equivalent Daily Dose

\begin{tabular}{lccccc} 
& 1 & 2 & 3 & 4 & 5 \\
\hline $\begin{array}{l}\text { Use alternative therapies } \\
\text { before pharmacologic therapy }\end{array}$ & $6.5(4)$ & $1.6(1)$ & $12.9(8)$ & $16.1(10)$ & $62.9(39)$ \\
Co-prescribe naloxone & $58.1(36)$ & $21(13)$ & $8.1(5)$ & $8.1(5)$ & $4.8(3)$ \\
Co-prescribe benzodiazepines & $38.7(24)$ & $40.3(25)$ & $17.7(11)$ & $1.6(1)$ & $1.6(1)$ \\
Prescribe over 50 MEDD & $37.1(23)$ & $33.9(21)$ & $12.9(8)$ & $8.1(5)$ & $8.1(5)$ \\
Prescribe over 90 MEDD & $50(31)$ & $29(18)$ & $8.1(5)$ & $4.8 .(3)$ & $8.1(5)$
\end{tabular}

these practices mandatory, requiring support in order to enforce them. Preliminary chart review at UTSW showed low adherence overall.

Objectives Our aim was to improve opioid treatment standards in CNCP patients at UTSW by increasing the number of physicians adhering to Texas Medical Board CNCP treatment requirements to $100 \%$ by December 2020.

Methods A three-part intervention including an electronic medical record (EMR) navigator tool (figure 1), chronic opioids registry, and physician education was developed to improve accessibility, documentation, and understanding of opioid prescribing guideline recommendations. A survey was developed to measure physician barriers to policy adherence, attitudes toward the policy components, and current opioid prescribing practices.
Results Physicians who had used the EMR navigator tool reported overall greater use of several guideline-concordant treatment components compared to those who had not used it (figure 2). Physicians who received opioid prescribing training were more aware and familiar with the policy. Those who had used the EMR navigator tool were more likely to implement pain management agreements and check the PMP (table 1). Only a small percentage of respondents reported co-prescribing naloxone for high-risk CNCP individuals (table 2).

Conclusions An EMR navigator tool to improve accessibility of treatment components is effective in improving policy adherence at our institution. These interventions may serve as a valuable tool at other academic medical centers to support safer opioid prescribing and provide a framework for tools to support other guidelines as well.

\section{REDUCING CENTRAL LINE ASSOCIATED BLOODSTREAM INFECTIONS IN THE NEUROSURGICAL AND SURGICAL INTENSIVE CARE UNITS}

Geralda Xavier, Briana Episcopia, Mikael Phillip. NYC Health + Hospitals/Kings County, USA

\subsection{6/bmjoq-2020-IHI.26}

Background In 2018 there was an increase in Central Line Associated Bloodstream Infections (CLABSIs) in the Neurosurgical and Surgical Intensive Care Units at NYC Health + Hospitals/Kings County

Objectives We initiated a CLABSI reduction project with a goal to reduce the CLABSI rate by $50 \%$ by the end of 2019 , which was achieved.

Methods Through root cause analysis (RCA) of patients with central line catheters, CLABSI event data was analyzed and a special cause variation was revealed in patients on total parenteral nutrition (TPN). Retrospective review of data from January 2017 - March 2019 revealed 7 TPN-related candidemias. An interdisciplinary team was assembled to perform multiple tests of change. Using point prevalence data, we observed a $47 \%$ bundle compliance rate at the project's onset, with the initial PDSA cycle focusing on bundle adherence for line maintenance. After implementing and sustaining our initial PDSA cycle, we focused additional cycles to included empiric treatment with fluconazole for patients on TPN over 7 days and implementation of dedicated ICU multidisciplinary care team rounds.

Results Following these improvement cycles, we reduced our total CLABSI rate by $50 \%$ and we saw 0 TPN-related candidemia over 10 consecutive months. From 2018-2019, our overall CLABSI rate in the ICU decreased from 3.6 to 1.8 per 1000 central line days. Using the Association for Professionals in Infection Control and Epidemiology (APIC) Cost of Healthcare-Associated Infections Model we estimate a $\$ 100,854$ cost savings and 51 avoided excess hospital days in 2019.

Conclusions Through focused unit-based education, implementation of a standardized bundle compliance monitoring tool and capture/circulation of staff feedback, we saw rates improve to $98 \%$, which has sustained over time. We observed patients on TPN greater than 7 days are most susceptible to infection. However, multidisciplinary rounds with shared accountability improved compliance with bundle. 